\section{Case Reports in Ophthalmology}

Case Rep Ophthalmol 2017;8:521-526

DOI: $10.1159 / 000484918$

Published online: November 17, 2017
(C) 2017 The Author(s)

Published by S. Karger AG, Basel www.karger.com/cop

This article is licensed under the Creative Commons Attribution-NonCommercialNoDerivatives $4.0 \quad$ International License (CC BY-NC-ND) (http://www.karger.com/Services/OpenAccessLicense). Usage and distribution for commercial purposes as well as any distribution of modified material requires written permission.

\title{
Baerveldt Scleral Patch Graft Abscess Secondary to Coagulase-Negative Staphylococcus
}

\author{
Van Nguyen ${ }^{a} \quad$ Mason Schmutz $^{b}$ Sarah Farukhic Sameh Mosaed ${ }^{d}$ \\ ${ }^{a}$ UCI Department of Internal Medicine, Orange, CA, USA; ${ }^{b}$ UCI Department of \\ Ophthalmology, Orange, CA, USA; ' UCI Department of Ophthalmology, Orange, CA, USA; \\ dUCI Department of Ophthalmology, Glaucoma Faculty, Orange, CA, USA
}

\section{Keywords}

Glaucoma Scleral patch graft $\cdot$ Abscess $\cdot$ Coagulase-negative Staphylococcus

\begin{abstract}
We report the case of a 69-year-old female who underwent a Baerveldt implant placement for severe-stage primary open-angle glaucoma and developed a bacterial infection of the conjunctiva and abscess of the scleral patch graft with subsequent tube exposure. The infection was identified 3 weeks postoperatively and a topical antibiotic was immediately initiated. A concurrent systemic staphylococcal infection was discovered by an outside physician and oral cephalexin was initiated. Despite antibiotic treatment, the conjunctival erosion progressed, and tube revision was required. Culture of the abscess revealed coagulase-negative Staphylococcus. As alluded to above, the patient also had multiple abscesses on the skin that cultured positive for Staphylococcus aureus. To our knowledge, this is the first case in the literature of coagulase-negative Staphylococcus causing an early postoperative abscess of the scleral patch graft following glaucoma drainage device placement.
\end{abstract}




\section{Case Reports in Ophthalmology}

Nguyen et al.: Baerveldt Scleral Patch Graft Abscess Secondary to Coagulase-Negative Staphylococcus

\section{Introduction}

Glaucoma is the leading cause of irreversible blindness in the world [1]. Glaucoma treatment is aimed at lowering intraocular pressure (IOP) in an effort to prevent further damage to the optic nerve and slow down progression of visual field loss damage. IOP can be lowered with medications, laser procedures, or incisional surgeries. Glaucoma drainage devices (GDD), such as the Ahmed valve and Baerveldt implant are often utilized after more conservative treatments have failed [2].

Known complications of GDD surgery include intraocular bleeding, choroidal detachment, erosion of the conjunctiva, hypotony, corneal decompensation, strabismus, tube obstruction, tube exposure, and infection $[3,4]$.

Risk factors for tube exposure include smoking, pseudoexfoliation glaucoma, history of dry eye syndrome, diabetes, younger age, and inflammation [5, 6]. Sclera, pericardium, cornea, and dura mater are the commonly used graft materials to cover a GDD [7]. The most significant complication related to exposure is endophthalmitis, with a reported range from 0.8 to $6.3 \%$ and a mean of $2 \%$ [8]. Our literature search in June 2017 via PubMed revealed no research that detailed frequencies or common causes of external infections of the conjunctiva and patch graft following GDD surgery.

\section{Case Description}

Our patient is a 69-year-old female with a history of severe-stage primary open-angle glaucoma refractory to previous trabeculectomy and on maximum topical medical treatment in the left eye. She underwent placement of a Baerveldt glaucoma implant with a scleral patch graft (SPG) in the left eye with vicryl suture tube ligature. The surgery was unremarkable and without complications. On postoperative day 1 , she was doing well, the tube was in good position, and her incision was closed with no exposure. Her IOP was $34 \mathrm{~mm} \mathrm{Hg}$, and her best corrected visual acuity (BCVA) was 20/70 in the left eye. She was treated with our routine postoperative regimen of moxifloxacin 4 times a day for 1 week and prednisolone acetate starting at 4 times a day and tapered weekly. She was also placed on her preoperative glaucoma drops, while awaiting ligature dissolution. At postoperative week 3 , the patient reported irritation and discharge in the left eye and was noted to have an abscess and erosion of underlying conjunctiva and partial erosion of the SPG. Her vision was slightly improved with a BCVA of 20/60 and an IOP of $14 \mathrm{~mm} \mathrm{Hg}$ at this time. A mucoid, pigmented plaque from the abscess was sent for culture. The patient was restarted on moxifloxacin 4 times a day.

Culture results were finalized as coagulase-negative Staphylococcus, susceptible to gentamicin. She was started on gentamicin, and moxifloxacin was continued. At the follow-up visit, slit-lamp examination revealed an enlarging area of thinning and erosion of the SPG with overlying avascular conjunctiva with a central area of eroded conjunctiva. There was no infiltrate and no signs of active infection. The wound was Seidel negative with no exposure of the tube.

It was at this visit that the patient reported developing a rash involving her back, right arm, and abdomen with scattered abscesses. She went to a walk-in clinic 1 week prior and was initially started on topical steroids for the skin infection while waiting for culture results which later grew Staphylococcus aureus. Topical steroid was reportedly stopped and cephalexin was started by the urgent care physician. 
At her subsequent follow-up a week later, the erosion of the conjunctiva and SPG had progressed with exposure of the glaucoma implant (Fig. 1). The patient was scheduled for wound revision with removal of the SPG, conjunctivoplasty, and placement of a new SPG (Fig. 2). The old SPG and surrounding conjunctiva were sent for culture and showed no growth of bacteria or fungus.

At postoperative week 6 after the revision, the tube remained covered with no recurrence of erosion or infection, and her skin lesions resolved on the regimen of cephalexin.

\section{Discussion}

Infection occurs in roughly $1 \%$ of GDD procedures and can occur months to years after the initial operation, sometimes requiring shunt removal; of those infections, most cases are forms of endophthalmitis [9]. In a retrospective study of Ahmed glaucoma valve implant complications in 542 eyes of 505 patients, endophthalmitis occurred in 1.7\% of patients, with the most common organisms including Streptococcus species and Haemophilus influenzae [10].

Graft exposure is a major risk factor for infection [9]. In a recent retrospective case series of 763 cases comparing graft materials, exposure for SPGs was lowest (0.5\%) compared to pericardium (7.9\%) and cornea (9.2\%), although it approached but did not reach statistical significance ( $p=0.072)$ [9]. The following studies have different rates of tube exposure for the various graft materials, but a far smaller sample size. In one study of 64 eyes comparing sclera, dura, and pericardium patch grafts, exposure rates were $4.3,5.6$, and $0 \%$, respectively [11]. Regarding pericardial grafts in another study, the exposure rate was $16 \%$ with single-thickness processed pericardium in 31 eyes compared to $0 \%$ in 59 eyes covered with double-thickness processed pericardium $(p=0.0002)$ [12]. A separate study looking at corneal patch grafts in 45 eyes noted a tube exposure frequency of $2.2 \%$ [13], while another looking at VisionGraft gamma-irradiated cornea had an exposure rate of $1.8 \%$ in 169 eyes [14]. The sample sizes of the latter studies were far smaller than that of the first study, which suggests a slight advantage for SPG in regards to tube exposure, but it is reasonable to believe that double-thickness processed pericardium and gamma-irradiated cornea may also reduce risk of exposure and possible subsequent infection.

Our literature search yielded many studies looking at endophthalmitis in the postoperative period, with a paucity of reports of focal, external infections of the conjunctiva or the patch graft, and no reported cases of that kind of infection due to coagulase-negative Staphylococcus leading to an abscess. In a cross-sectional study of ocular infections unrelated to GDD placement, $S$. aureus was the leading cause of conjunctivitis $(21.5 \%)$ with coagulasenegative Staphylococcus as the second leading cause (16.5\%) [15]. We found one case of an external infection in a patient after GDD surgery for neovascular glaucoma, which was due to methicillin-resistant S. aureus [16].

Our patient had a conjunctival pigmented mucoid plaque with underlying abscess formation and conjunctival melt following an uneventful GDD placement. Typically, erosions lead to underlying infections, but in this case, it was the reverse. Our patient's SPG infection was due to coagulase-negative Staphylococcus infection most likely due to a foreign body which caused colonization and involvement of the ocular surface. That strain is common in nosocomial infections and frequently related to foreign bodies. Another possibility is that the SPG itself may have carried infection preoperatively. Given that the two strains were different, it is unlikely that it was a systemic infection that manifested in the eye. Her concurrent 
wound infection and skin infection, however, are curious and may have been due to some predisposing systemic vulnerability.

There are 2 disease entities that lead to recurrent Staphylococcus abscesses: chronic granulomatous disease and hyper IgE syndrome (i.e., Job syndrome). However, these usually present early in life and have other presenting features such as short stature, chronic diarrhea, and gingivitis for chronic granulomatous disease and coarse facies, retained primary teeth, and eczema for hyper IgE syndrome, none of which were present in our patient. Given the very low likelihood of these conditions, we did not pursue further testing.

Clinicians must be aware of conditions that could lead to poor postoperative outcomes such as concurrent staphylococcal skin infection, as in our patient. At-risk patients may benefit from alternative nonincisional therapies such as transscleral diode cyclophotocoagulation, which does not have an associated infection risk and has shown to have similar outcomes at 2 years when compared to Ahmed glaucoma valve in patients with neovascular glaucoma [17]. Known Staphylococcus carriers or those predisposed to infection by Staphylococcus species may benefit from preoperative mupirocin treatment, chlorhexidine baths, the use of antibiotics known to have a high sensitivity to respective strains, and avoidance of those antibiotics with high resistance, such as fluoroquinolones [18]. Patients with higher risk of infection would also benefit from SPGs, gamma-irradiated cornea, or double-thickness pericardium $[11,12,14]$. Surgical and nonsurgical options, graft material, and perioperative treatment should be based on patient characteristics, underlying predisposing conditions, and known bacterial colonization.

\section{Statement of Ethics}

This case report complies with the guidelines for human studies and animal welfare regulations. No personal health identifiers have been included, and per the protocol of the institutional review board at University of California, Irvine, any case series with less than three (3) subjects do not require approval by the institution's committee on human research.

\section{Disclosure Statement}

None of the authors have any proprietary interests or conflicts of interest related to this submission. This submission has not been published anywhere previously and is not simultaneously being considered for any other publication.

\section{References}

1 Quigley HA, Broman AT: The number of people with glaucoma worldwide in 2010 and 2020. Br J Ophthalmol 2006;90:262-267.

2 Jonas JB, Aung T, Bourne RR, Bron AM, Ritch R, Panda-Jonas S: Glaucoma. Lancet 2017;S01406736(17)31469-1.

3 Mandalos A, Sung V: Glaucoma drainage device surgery in children and adults: a comparative study of outcomes and complications. Graefes Arch Clin Exp Ophthalmol 2017;255:1003-1011.

-4 Riva I, Roberti G, Oddone F, Konstas AG, Quaranta L: Ahmed glaucoma valve implant: surgical technique and complications. Clin Ophthalmol 2017;11:357-367.

-5 Netland P, Chaku M, Ishida K, Rhee D: Risk factors for tube exposure as a late complication of glaucoma drainage implant surgery. Clin Ophthalmol 2016;10:547-553. 


\section{Case Reports in \\ Ophthalmology}

\begin{tabular}{l|l}
\hline DOI: $10.1159 / 000484918$ & ( 2017 The Author(s). Published by S. Karger AG, Basel \\
\hline
\end{tabular}
www.karger.com/cop

Nguyen et al:: Baerveldt Scleral Patch Graft Abscess Secondary to Coagulase-Negative Staphylococcus

6 Trubnik V, Zangalli C, Moster MR, et al: Evaluation of risk factors for glaucoma drainage device-related erosions: a retrospective case-control study. J Glaucoma 2015;24:498-502.

-7 Thakur S, Ichhpujani P, Kumar S: Grafts in glaucoma surgery: a review of the literature. Asia Pac J Ophthalmol (Phila) 2017;6:469-476.

-8 Gedde SJ, Scott IU, Tabandeh H, et al: Late endophthalmitis associated with glaucoma drainage implants. Ophthalmology 2001;108:1323-1327.

-9 Levinson JD, Giangiacomo AL, Beck AD, et al: Glaucoma drainage devices: risk of exposure and infection. Am J Ophthalmol 2015;160:516-521.e2.

$\rightarrow 10$ Al-Torbak AA: Endophthalmitis associated with the Ahmed glaucoma valve implant. Br J Ophthalmol 2005;89:454-458.

11 Smith MF, Doyle JW, Ticrney JW Jr: A comparison of glaucoma drainage implant tube coverage. J Glaucoma 2002;11:143-147.

12 Lankaranian D, Reis R, Henderer JD, Choe S, Moster MR: Comparison of single thickness and double thickness processed pericardium patch graft in glaucoma drainage device surgery: a single surgeon comparison of outcome. J Glaucoma 2008;17:48-51.

13 Spierer 0, Waisbourd M, Golan Y, Newman H, Rachmiel R: Partial thickness corneal tissue as a patch graft material for prevention of glaucoma drainage device exposure. BMC Ophthalmol 2016;16:20

14 Ekici F, Moster MR, Cvintal V, Hu WD, Waisbourd M: Tube shunt coverage with gamma-irradiated cornea allograft (VisionGraft). Clin Ophthalmol 2015;9:751-755.

15 Teweldemedhin M, Saravanan M, Gebreyesus A, Gebreegziabiher D: Ocular bacterial infections at Quiha Ophthalmic Hospital, Northern Ethiopia: an evaluation according to the risk factors and the antimicrobial susceptibility of bacterial isolates. BMC Infect Dis 2017;17:207.

-16 Pansegrau ML, Mengarelli E, Dersu II: Complication of an Ahmed glaucoma valve implant: tube exposure with methicillin-resistant Staphylococcus aureus infection. Digit J Ophthalmol 2015;21:1-9.

$\checkmark 17$ Yildirim N, Yalvac IS, Sahin A, Ozer A, Bozca T: A comparative study between diode laser cyclophotocoagulation and the Ahmed glaucoma valve implant in neovascular glaucoma: a long-term follow-up. J Glaucoma 2009;18:192-196.

18 Miller D: Update on the Epidemiology and Antibiotic Resistance of Ocular Infections. Middle East Afr J Ophthalmol 2017;24:30-42.

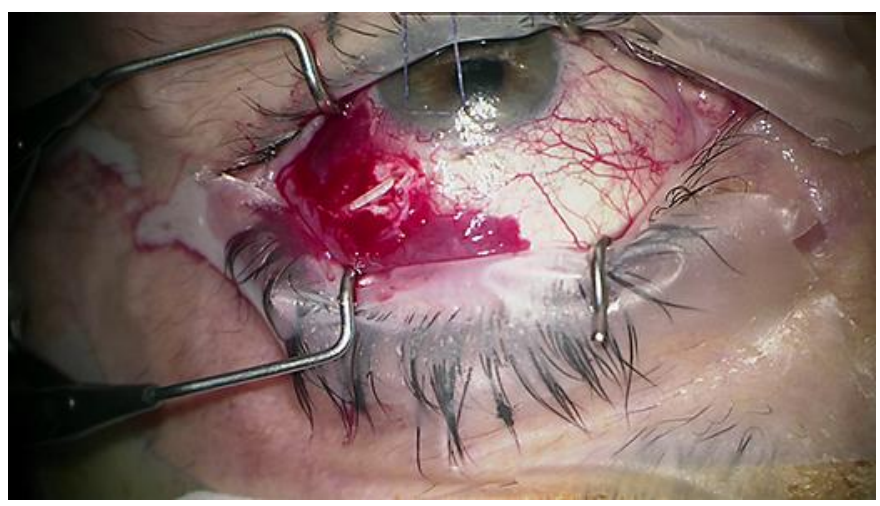

Fig. 1. The erosion is radial, which suggests that it is unrelated to the circumferential incision. 

www.karger.com/cop

Nguyen et al.: Baerveldt Scleral Patch Graft Abscess Secondary to Coagulase-Negative Staphylococcus

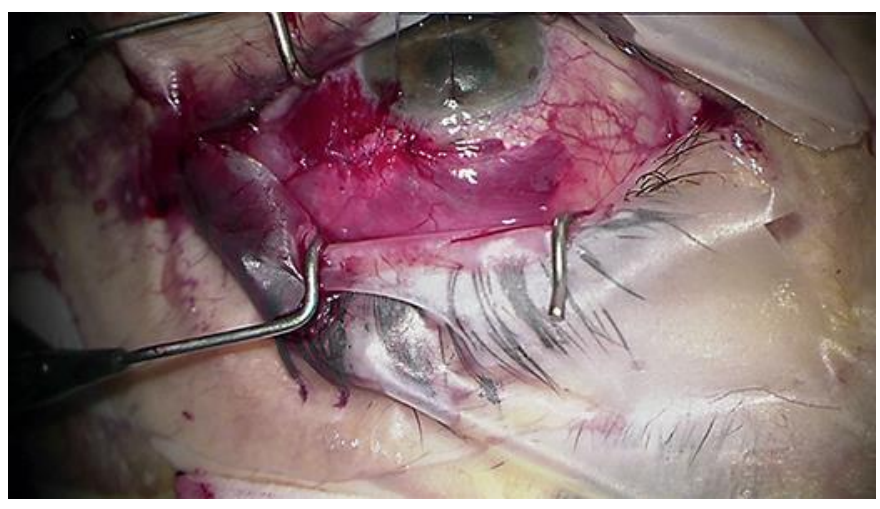

Fig. 2. The conjunctiva closed over the replacement scleral patch graft. 